\title{
Hyper-Non-Gaussianities in Inflation with Strongly Nongeodesic Motion
}

\author{
Jacopo Fumagalli, Sebastian Garcia-Saenz, Lucas Pinol, Sébastien Renaux-Petel@, and John Ronayne \\ Institut d'Astrophysique de Paris, GReCO, UMR 7095 du CNRS et de Sorbonne Université, \\ 98 bis boulevard Arago, Paris 75014, France
}

(Received 6 March 2019; revised manuscript received 25 October 2019; published 15 November 2019)

\begin{abstract}
Several recent proposals to embed inflation into high-energy physics rely on inflationary dynamics characterized by a strongly nongeodesic motion in negatively curved field space. This naturally leads to a transient instability of perturbations on sub-Hubble scales, and to their exponential amplification. Supported by first-principles numerical computations, and by the analytical insight provided by the effective field theory of inflation, we show that the bispectrum is enhanced in flattened configurations, and we argue that an analogous result holds for all higher-order correlation functions. These "hyper-nonGaussianities" thus provide powerful model-independent constraints on nonstandard inflationary attractors motivated by the search for ultraviolet completions of inflation.
\end{abstract}

DOI: 10.1103/PhysRevLett.123.201302

Introduction.-Negatively curved field space plays a crucial role in modern embeddings of inflation in highenergy physics. Nonlinear sigma models with a hyperbolic target space arise naturally in top-down realizations of inflation, particularly within supergravity, giving rise to the $\alpha$-attractor class of models (see, e.g., Refs. [1-3]). Independently of the question of their ultraviolet completions, nonminimal kinetic terms of the hyperbolic type lead to interesting dynamics, allowing for nontrivial inflationary trajectories characterized by a strongly nongeodesic motion [4-7]. This in turn relaxes the conditions of slow-roll to allow for potentials that are steep in Planck units [8,9], a welcome feature in view of the eta problem and the recently much discussed swampland conjectures [10-12]. Lastly, internal field spaces with negative curvature are at the origin of the phenomenon of geometrical destabilization [13-17], in which noninflationary degrees of freedom, even heavy ones, can dramatically affect the fate of inflation.

A concrete scenario in which the consequences of a hyperbolic field space have been studied is the proposal of "hyperinflation" [18], which has recently been under scrutiny $[19,20]$. The intuitive picture of this setup is that of an inflationary trajectory corresponding to a circular motion around the minimum of a (circularly symmetric) scalar potential. The hyperbolic geometry is crucial to compensate for the loss of angular velocity to the Hubble friction, allowing inflation to last long enough, even if the potential is too steep to inflate along a radial trajectory.

Published by the American Physical Society under the terms of the Creative Commons Attribution 4.0 International license. Further distribution of this work must maintain attribution to the author(s) and the published article's title, journal citation, and DOI. Funded by SCOAP ${ }^{3}$.
Within this circumstance, hyperinflation proceeds along a strongly nongeodesic trajectory, and a striking outcome is an exponential growth of the curvature power spectrum around the time of Hubble crossing, and the corresponding suppression of the tensor-to-scalar ratio. With such an amplification, assessing the size of nonlinear effects in this setup appears to be crucial, while previous studies have restricted their attention to the analysis of linear fluctuations.

In this context, this Letter presents a general framework to study non-Gaussianities in the presence of strongly nongeodesic motion typical of hyperbolic-type geometry, highlighting how this naturally leads to "hyper-nonGaussianities." For definiteness we concentrate on the specific example of hyperinflation as a particularly interesting playground to analyze the effects of the nontrivial field space in this class of models. However our results are formulated in general terms and have a broad range of applicability. Essentially, they indicate that in negatively curved field space, inflationary models with strongly nongeodesic motion are characterized by an enhanced nonGaussian signal, both for the bispectrum and for all higherpoint correlation functions, that can easily lead to tensions with experimental bounds. These model-independent constraints sharpen the range of allowed theoretical constructions, and are of utmost importance in view of the intense current efforts to build nonstandard inflationary scenarios in agreement with quantum gravity conjectures.

Hyperinflation.-The starting point is an action for two scalar fields $\varphi^{I}=(\phi, \theta)$ with noncanonical kinetic term minimally coupled to gravity:

$S=\int d^{4} x \sqrt{-g}\left(\frac{M_{\mathrm{Pl}}^{2}}{2} R-\frac{1}{2} G_{I J} \nabla^{\mu} \varphi^{I} \nabla_{\mu} \varphi^{J}-V(\varphi)\right)$. 
The matrix $G_{I J}$ defines a metric in the internal field space parametrized by the coordinates $\varphi^{I}$, in this case the hyperbolic plane of curvature $-2 / M^{2}$, and is assumed to have the form

$$
G_{I J} d \varphi^{I} d \varphi^{J}=d \phi^{2}+M^{2} \sinh ^{2}\left(\frac{\phi}{M}\right) d \theta^{2}
$$

Moreover, the potential is assumed to depend only on the "radial" field $\phi, V=V(\phi)$, with $V^{\prime}>0$.

Consider now an inflationary background characterized by homogeneous fields $\phi(t)$ and $\theta(t)$, and a quasi-de Sitter spacetime metric with scale factor $a(t)$ and Hubble parameter $H(t)=\dot{a} / a$, with $t$ the cosmological time. Hyperinflation corresponds to a nonstandard attractor solution of the action (1), with small parameters $\epsilon \equiv$ $-\dot{H} / H^{2} \simeq 3 M V^{\prime} / 2 V$ and $\eta \equiv \dot{\epsilon} / H \epsilon \simeq 2 \epsilon-3 M V^{\prime \prime} / V^{\prime}$, that arises under the conditions

$$
\frac{3 M}{M_{\mathrm{Pl}}}<\frac{M_{\mathrm{Pl}} V^{\prime}}{V} \ll \frac{M_{\mathrm{Pl}}}{M}, \quad \frac{M\left|V^{\prime \prime}\right|}{V^{\prime}} \ll 1 .
$$

More precisely, the equation of motion $\ddot{\phi}+3 H \dot{\phi}-$ $M \sinh (\phi / M) \cosh (\phi / M) \dot{\theta}^{2}+V^{\prime}(\phi)=0$ admits a solution with $\dot{\phi} \simeq-3 M H$, independently of the slope of the potential. Defining $h^{2} \equiv\left[V^{\prime}(\phi) / M H^{2}\right]-9$, a positive quantity for hyperinflation solutions [see Eq. (3)], one has $h^{2} / 9+1 \simeq \epsilon_{V} / \epsilon \simeq \eta_{V} /(2 \epsilon-\eta)$, where $\epsilon_{V}=$ $\frac{1}{2} M_{\mathrm{Pl}}^{2}\left(V^{\prime} / V\right)^{2}$ and $\eta_{V}=M_{\mathrm{Pl}} V^{\prime \prime} / V$ are the standard potential "slow-roll" parameters, not necessarily small here. In hyperinflation, potentials that verify the swampland de Sitter conjecture (in its refined version [21,22]) should obey either $\epsilon_{V} \geq \mathcal{O}(1)$ or $-\eta_{V} \geq \mathcal{O}(1)$, corresponding, respectively, to a steep slope or steep negative curvature in Planck units. From the above relations, one deduces that a prolonged phase of hyperinflation supported by such potentials is necessarily characterized by $h^{2} \gg 1$ (see also Ref. [20]). We will concentrate on this theoretically most interesting regime, which, as we will see, corresponds to a strongly nongeodesic motion. Actually, as emphasized recently in a model-independent manner [9], the latter feature is necessary in order to inflate on potentials whose slope is steep in Planck units, and it is also a characteristic feature of the sidetracked models studied in Ref. [6].

Strongly nongeodesic motion and dynamics of linear perturbations.-Let us now consider linear fluctuations. We employ gauge invariant variables $Q^{I}$ that coincide with the field fluctuations $\delta \varphi^{I}$ in the spatially flat gauge, and perform a decomposition in terms of the adiabatic and entropic modes $Q_{\sigma}$ and $Q_{s}$, defined as the projection of $Q^{I}$ in the direction tangential and perpendicular to the background trajectory respectively, the inner product being defined by the field space metric $G_{I J}$ (see, e.g., Ref. [23] for a review of perturbation theory in multifield inflation). The adiabatic mode can be expressed as
$Q_{\sigma}=(\dot{\sigma} / H) \zeta$, with the definition $\dot{\sigma} \equiv \sqrt{G_{I J} \dot{\varphi}^{I} \dot{\varphi}^{J}}$, and where $\zeta$ is the comoving curvature perturbation. The equation of motion for the entropic perturbation is given by

$$
\ddot{Q}_{s}+3 H \dot{Q}_{s}+\left(\frac{k^{2}}{a^{2}}+m_{s}^{2}\right) Q_{s}=-2 \dot{\sigma} \eta_{\perp} \dot{\zeta},
$$

where $k$ is the Fourier wave number, we introduced

$\eta_{\perp} \equiv-\frac{V_{, s}}{H \dot{\sigma}}, \quad m_{s}^{2} \equiv V_{; s s}-H^{2} \eta_{\perp}^{2}+\epsilon H^{2} R_{\mathrm{fs}} M_{\mathrm{Pl}}^{2}$.

Here $V_{, s}$ and $V_{; s s}$ stand for the projections in the entropic direction of the first and second (field-space covariant) derivatives of the potential, respectively, and $R_{\mathrm{fs}}$ is the field space scalar curvature. The "bending" parameter $\eta_{\perp}$ is physically important as it gives a measure of the deviation of the background trajectory from a geodesic in field space [24]. A strongly nongeodesic motion is characterized by $\eta_{\perp}^{2} \gg 1$, resulting in a large negative contribution to the entropic mass $m_{s}^{2}$, something that a negatively curved field space only reinforces. Without a stabilization from the potential, a large negative $m_{s}^{2} / H^{2}$ is thus a built-in feature of these models. Although unusual, this property is not a priori in contradiction with the requirement of a stable background. Indeed, on super-Hubble scales $k / a \ll H$ one has $\dot{\zeta}=2 H^{2} \eta_{\perp} / \dot{\sigma} Q_{s}$, and (4) yields an uncoupled equation for $Q_{s}$, now with a different effective mass $m_{s(\text { eff })}^{2} \equiv$ $m_{s}^{2}+4 H^{2} \eta_{\perp}^{2}$, to which the bending contributes positively.

Specifying this general discussion to hyperinflation, we find, to leading-order in the slow-varying approximation,

$$
\eta_{\perp}^{2} \simeq h^{2}, \quad m_{s}^{2} \simeq-2 H^{2} h^{2}, \quad m_{s(\mathrm{eff})}^{2} \simeq 2 H^{2} h^{2} .
$$

As anticipated, a strongly nongeodesic motion corresponds to $h^{2} \gg 1$. In this situation, the large and positive $m_{s(\text { eff })}^{2} / H^{2}$ implies a rapid decay of $Q_{s}$ on super-Hubble scales and the conservation relation $\dot{\zeta} \simeq 0$. The decay of homogeneous perturbations $Q_{s}(k=0)$ is a proof that the background solution of hyperinflation is indeed a stable attractor. On the contrary, the large and negative $m_{s}^{2} / H^{2}$ signals a transient instability of fluctuations on sub-Hubble scales, observed also in some sidetracked inflationary models [6] and earlier in Refs. [25,26]. These considerations can be checked numerically. In Fig. 1 we consider the same background as studied in Ref. [19], for the quadratic potential $V(\phi)=\frac{1}{2} m^{2} \phi^{2}$ with $m=M=10^{-2} M_{\mathrm{Pl}}$. We display the time dependence of the dimensionless curvature $\left(\mathcal{P}_{\zeta}\right)$ and entropic $\left(\mathcal{P}_{\mathcal{S}}=H^{2} / \dot{\sigma}^{2} \mathcal{P}_{Q_{s}}\right)$ power spectra, for the scale $k_{55}$ that crosses the Hubble radius $55 e$-folds before the end of inflation, which we take as the $\mathrm{CMB}$ pivot scale. The normalization factor is the "standard" result $\mathcal{P}_{0}=H^{2} /$ $\left(8 \pi^{2} \epsilon M_{\mathrm{Pl}}^{2}\right)_{k_{55}=a H}$. Soon after "entropic mass crossing" the exponential growth of entropic fluctuations caused by the 


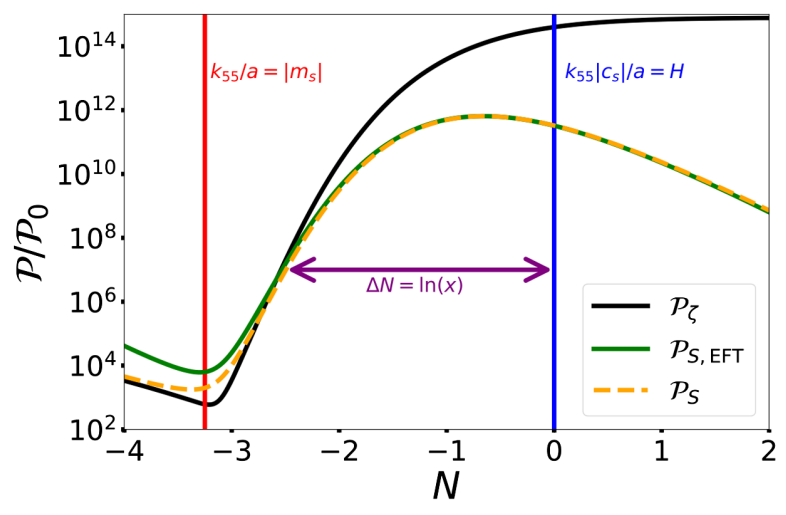

FIG. 1. Adiabatic $\left(\mathcal{P}_{\zeta}\right)$ and entropic $\left(\mathcal{P}_{\mathcal{S}}\right)$ power spectra as functions of the number of $e$-folds, for the representative model detailed in the main text. The spectra are evaluated for the scale $k_{55}$ that crosses the Hubble radius $55 e$-folds before the end of inflation, at $N=0$ in the plot. $\mathcal{P}_{\mathcal{S} \text {,EFT }}$ is the entropic power spectrum deduced from the relation (8).

tachyonic instability feeds the adiabatic perturbation, before entropic fluctuations decay and the curvature perturbation becomes constant. The transient instability results in an exponentially small tensor-to-scalar ratio $r=3.6 \times 10^{-16}$.

Bispectrum.-We now turn to the numerical calculation of the bispectrum in hyperinflation. (See, e.g., Refs. [27,28] for recent reviews on primordial non-Gaussianities, and Ref. [29] for the most recent observational constraints from the Planck Collaboration.) The results have been obtained with PyTransport 2.0 [30,31], a code based on the transport approach to compute two- and three-point correlation functions in multifield models with curved field space (see also CppTransport [32-34]). Figure 2 is a plot of the reduced bispectrum $f_{N L}\left(k_{1}, k_{2}, k_{3}\right)$ for the same representative model as above, as a function of the variables $(\alpha, \beta)$ defined by $k_{1}=\left(3 k_{55} / 4\right)(1+\alpha+\beta), \quad k_{2}=\left(3 k_{55} / 4\right)$ $(1-\alpha+\beta)$, and $k_{3}=\left(3 k_{55} / 2\right)(1-\beta)$. The resulting bispectrum is quite unlike what is usually found in inflationary models with a Bunch-Davies vacuum state. In particular, hyperinflation generates a non-Gaussian signal that is peaked near flattened triangle configurations, i.e., the ones with $k_{1} \simeq k_{2}+k_{3}$ (the edges in Fig. 2), which is typical of excited initial states. Explicitly, we find

$$
f_{N L}^{\mathrm{eq}}=-2.0, \quad f_{N L}^{\mathrm{flat}}=53.8,
$$

where the two parameters simply denote the evaluation of the reduced bispectrum, respectively, at $k_{1}=k_{2}=k_{3}$ and at the representative flattened configuration $k_{2}=k_{3}=$ $k_{1} / 2$. Although we have presented results for the particular case of a quadratic potential and for a specific set of parameters, we remark that the qualitative outcome-a strong non-Gaussian signal in flattened configurations-is quite robust. We find, for instance, $f_{N L}^{\text {flat }} \simeq 25$ for a Starobinsky-type potential and $f_{N L}^{\text {flat }} \simeq 100$ for a quartic potential, while $f_{N L}^{\mathrm{eq}}=O(1)$ in each case.

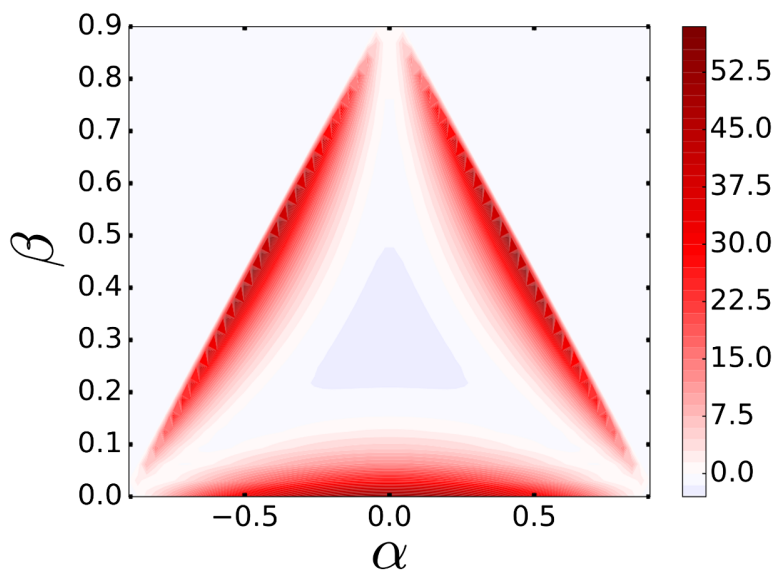

FIG. 2. Shape dependence $f_{N L}(\alpha, \beta)$, at fixed overall scale $k_{1}+k_{2}+k_{3}=3 k_{55}$, for the same model as in Fig. 1. The characteristic feature of the bispectrum is its dominant signal near flattened configurations. Note that the equilateral configuration corresponds to the point $(\alpha, \beta)=(0,1 / 3)$.

Effective single-field description.-In a standard setup with a single light degree of freedom, heavy entropic fluctuations can be integrated out to yield an EFT for the adiabatic mode (see, e.g., Refs. [25,35-38]). In the type of models we consider, the entropic field is heavy but tachyonic, yet the procedure can be carried out equivalently to the standard case, as explained in Ref. [39]. In slowly evolving backgrounds and in the regime $k^{2} / a^{2} \ll\left|m_{s}^{2}\right|$, one finds

$$
Q_{s}=-2 \frac{\dot{\sigma} \eta_{\perp}}{m_{s}^{2}} \dot{\zeta}
$$

which results in an effective quadratic action for the curvature perturbation:

$$
S_{\mathrm{eff}}^{(2)}=\int d \tau d^{3} x a^{2} \epsilon M_{\mathrm{Pl}}^{2}\left[\frac{\zeta^{\prime 2}}{c_{s}^{2}}-(\vec{\nabla} \zeta)^{2}\right],
$$

where $\tau \simeq-1 /(a H)$ is the conformal time, $\zeta^{\prime} \equiv d \zeta / d \tau$, and the speed of sound $c_{s}$ is defined by

$$
\frac{1}{c_{s}^{2}} \equiv 1+\frac{4 H^{2} \eta_{\perp}^{2}}{m_{s}^{2}}=\frac{m_{s(\mathrm{eff})}^{2}}{m_{s}^{2}} .
$$

An imaginary sound speed is thus a model-independent consequence of a tachyonic entropic mass $\left(m_{s}^{2}<0\right)$ and a stable background $\left(m_{s(\mathrm{eff})}^{2}>0\right)$. For instance, in hyperinflation we find $c_{s}^{2} \simeq-1$. In this class of models, the curvature perturbation thus propagates with a "wrong sign" dispersion relation: $\omega^{2}=-\left|c_{s}\right|^{2} k^{2} \simeq-k^{2}$. Accordingly, the mode functions do not oscillate but rather grow or decay exponentially, affecting all wave modes $k$ up to the cutoff of the EFT-what in the two-field theory was a tachyonic 
instability affecting low-energy modes has become in the EFT a gradient-type instability to which the whole spectrum is sensitive. Such a theory is not a priori catastrophic, as the corresponding instability is only transient. However, our results will ultimately bound the amplification of fluctuations.

It will prove useful to introduce the dimensionless parameter $x$ such that the wave modes $k$ described by the effective theory satisfy $k\left|c_{s}\right| / a<x H$. Its dependence on models' parameters is $x \sim\left|c_{s}\right|\left|m_{s}\right| / H$, where the numerical factor in the right should be somewhat smaller than unity. Quantitatively, $x$ can be determined by examining when the power spectrum of the entropic fluctuation computed in the full theory matches the one deduced from Eq. (8), which is the central relation from which the EFT derives. One can see in Fig. 1 that the two become in very good agreement less than one $e$-fold after entropic mass crossing, corresponding to $x \sim 10$ in this example. The general solution of the linear equation of motion reads, for $c_{s}^{2}<0$,

$$
\begin{aligned}
\zeta_{k}(\tau)= & \left(\frac{2 \pi^{2}}{k^{3}}\right)^{1 / 2} \alpha\left[e^{k\left|c_{s}\right| \tau+x}\left(k\left|c_{s}\right| \tau-1\right)\right. \\
& \left.-\rho e^{i \psi} e^{-\left(k\left|c_{s}\right| \tau+x\right)}\left(k\left|c_{s}\right| \tau+1\right)\right]
\end{aligned}
$$

where we omit the mild $k$ dependence of $(\alpha, \rho, \psi)$ for simplicity, and we stress that it only applies for $k\left|c_{s}\right| \tau+x \geq 0$. The parameter $\rho$ sets the relative amplitude of the exponentially decaying mode compared to the growing one at the time marking the validity of the EFT (and $\psi$ is a phase difference), while $\alpha$ can be taken to be real and parametrizes their overall amplitude. [The amplitude of the decaying mode is necessarily nonzero, as the quantization condition entails the relation $2 \alpha^{2} \rho \sin (\psi)\left|c_{s}\right|=\mathcal{P}_{0}$.] The final value of the curvature power spectrum $\mathcal{P}_{\zeta}=$ $\alpha^{2} e^{2 x} \quad$ [assuming very conservatively that $\rho \lesssim \mathcal{O}(1)$ ] depends on the initial conditions of the EFT. Although these can in principle be determined by matching to the full computation of $\mathcal{P}_{\zeta}(\tau)$, interestingly, this is not needed to study higher-order correlation functions, to which we now turn.

The cubic action of the EFT of inflationary perturbations $[40,41]$ reads (at lowest order in derivatives and in the slowvarying approximation):

$S_{\mathrm{eff}}^{(3)}=\int d \tau d^{3} x \frac{a \epsilon M_{\mathrm{Pl}}^{2}}{H}\left(\frac{1}{c_{s}^{2}}-1\right)\left[\zeta^{\prime}(\vec{\nabla} \zeta)^{2}+\frac{A}{c_{s}^{2}} \zeta^{\prime 3}\right]$,

where $A$ is a dimensionless constant of order 1 that can be computed from the full theory [42]. Although the interactions in Eq. (12) are standard, the behavior of the mode function (11) is not, and the computation of the bispectrum is nontrivial [39]. Contrary to what a naive power counting would indicate, one finds that the reduced bispectrum does not feature an exponential enhancement by $e^{2 x}$, like the power spectrum. Instead, it is independent of $x$ for near equilateral configurations, with

$$
f_{N L}^{\mathrm{eq}} \simeq \frac{10}{9}\left(\frac{1}{\left|c_{s}\right|^{2}}+1\right)\left(\frac{13 A}{6}-\frac{5}{24}\right) .
$$

Given the result $c_{s}^{2} \simeq-1$ in hyperinflation, we have the analytical prediction that $f_{N L}^{\mathrm{eq}}=O(1)$, in agreement with the numerical results. Away from the equilateral limit, one finds a dependence of $f_{N L}$ on the cutoff scale $x H$ (with the exception of the squeezed limit, which can be shown to verify the single-clock consistency relation via standard arguments). Akin to models with excited initial states (see, e.g., Refs. [43-47]) the constructive interferences between two growing and one decaying mode result in a magnification of the signal near flattened configurations $k_{1} \simeq k_{2}+k_{3}$,

$f_{N L}^{\text {flat }} \simeq \frac{1}{192}\left(\frac{1}{\left|c_{s}\right|^{2}}+1\right)\left[39(A-1)+12 x^{2}+4(A+1) x^{3}\right]$,

and a global shape with a large overlap with the orthogonal template (except for values of $A \simeq-1$ ) [39]. With $x^{2} \sim$ $h^{2} \gg 1$ in hyperinflation with strongly nongeodesic motion (e.g., $x^{2} \sim 100$ in our example), we conclude that flattened non-Gaussianities are large in this type of model, again in agreement with the full two-field numerical results (see the Supplemental Material [48] for a quantitative comparison).

Higher-order correlation functions.-We have seen that the single-field effective theory of perturbations with imaginary sound speed can unambiguously predict the striking features of the reduced bispectrum, thus providing a valuable device to gain analytical insight into the complex multifield dynamics of models with large negative entropic mass. But from a pragmatic perspective, the real power of the EFT approach is that it can go beyond the reach of current numerical methods, as we now show by providing an estimate of higher-order correlation functions in this class, including hyperinflation. Like for the bispectrum, for $n \geq 4$, the reduced connected $n$-point function $\left\langle\zeta^{n}\right\rangle /\left\langle\zeta^{2}\right\rangle^{n-1}$ does not feature an exponential amplification [49]. (This has been shown in Ref. [49] using the EFT put forward in the first version of this work.) In spite of this, we find that some flattened configurations for the trispectrum and higher-point correlators are enhanced by powers of the parameter $x$, essentially due to the same phenomenon of constructive interferences between growing and decaying modes that occurs for the bispectrum.

Focusing on the dominant contributions in the large $x$ limit, one can find an explicit derivation of the trispectrum in the Supplemental Material [48], where we also give further details of our estimate for the higher-point functions 


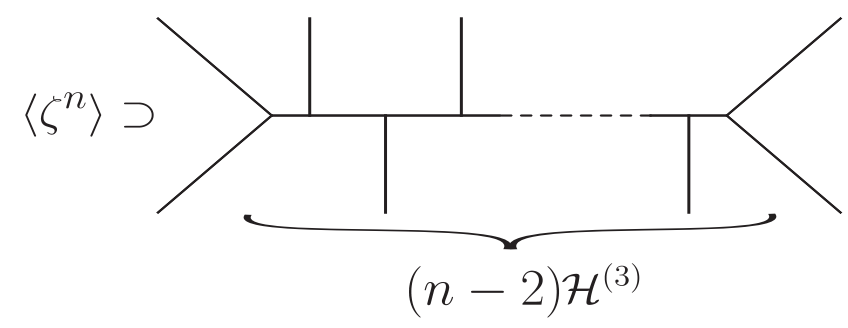

FIG. 3. Dominant contribution to the connected $n$-point correlation function of $\zeta$.

and characterize which flattened shapes are enhanced. Following the in-in formalism [50], the computation of the $n$-point correlator can be organized as a sum of connected Feynman diagrams with $n$ external lines, and with each insertion of the interaction Hamiltonian corresponding to a vertex. We find that a diagram with $v$ vertices contributes as $\left\langle\zeta^{n}\right\rangle /\left\langle\zeta^{2}\right\rangle^{n-1} \propto x^{2 n+v-4}$ for the maximally enhanced flattened configurations. This contribution is largest when $v=n-2$, corresponding to diagrams of the type shown in Fig. 3, with $(n-2)$ insertions of the cubic Hamiltonian, yielding the estimate

$$
\frac{\left\langle\zeta^{n}\right\rangle}{\left\langle\zeta^{2}\right\rangle^{n-1}} \sim\left[\left(\frac{1}{\left|c_{s}\right|^{2}}+1\right) x^{3}\right]^{n-2} .
$$

Notice that, despite the rapid growth of the correlation functions as $n$ increases, the theory is nevertheless under perturbative control for observationally relevant models. Indeed, perturbativity is guaranteed provided $\left\langle\zeta^{n}\right\rangle /\left\langle\zeta^{2}\right\rangle^{n-1} \mathcal{P}_{\zeta}^{(n-2) / 2} \sim\left(f_{N L}^{\text {flat }} A_{s}^{1 / 2}\right)^{n-2} \lesssim 1$, which is a weaker requirement than meeting the observational bounds on the bispectrum.

Discussion.--In negatively curved field space, and in the absence of a stabilizing effect from the potential in the direction perpendicular to the background trajectory, a strongly nongeodesic motion in field space automatically induces a transient instability of fluctuations on sub-Hubble scales. Under these general circumstances, we can make use of an effective field theory for the curvature perturbation that naturally explains the exponential amplification of the power spectrum. Moreover, it predicts a reduced bispectrum whose characteristics are in striking agreement with first-principles numerical computations in the full theory: order-one non-Gaussianities in equilateral configurations, and a magnification near flattened ones. However, contrary to the power spectrum, the reduced bispectrum is not exponentially amplified. We have moreover argued that an analogous outcome holds for all higher-order reduced correlation functions, namely, a hierarchical enhancement for particular flattened shapes proportional to a power of the instability rate. When the latter is very large, these hyper-non-Gaussianities lead to tensions with observational constraints, as exemplified by models of hyperinflation that satisfy the de Sitter swampland conjecture.

Our model-independent results severely bound the magnitude of a large negative entropic mass. Hence, it results in a powerful selection criterion on models with negatively curved field space and a strongly nongeodesic motion that have been receiving much attention recently. Namely, at least some stabilizing effect by the potential is needed to counterbalance the otherwise strongly tachyonic mass of entropic fluctuations on sub-Hubble scales. This compensating effect can be large, to the extent that $\left|m_{s}^{2} / H^{2}\right| \ll 1$ or $m_{s}^{2} / H^{2} \gg 1$, which are well understood situations. It can also be mild, resulting again in a negative entropic mass, but not parametrically larger than the Hubble scale (a feature shared by hyperinflation with a moderate degree of bending). Without hierarchy, an EFT cannot be rigorously derived, but we expect our results to give a qualitatively correct picture, i.e., an enhancement of the bispectrum in flattened configurations, and similarly for the trispectrum. Thus, our results pave the way for future studies about the role of field-space geometry in the dynamics of inflation, and particularly in how nongeodesic motion of inflationary attractors can lead to novel signatures that can be probed with current and next-generation experiments.

We are very grateful to D. Andriot, T. Bjorkmo, P. Christodoulidis, A. Joyce, D. Marsh, S. Mizuno, S. Mukohyama, A. Nicolis, E. Pajer, D. Roest, and K. Turzyński for interesting and helpful discussions, and to the anonymous referees that helped improving our Letter. J. F, L. P, S. R-P., and J. R. are supported by the European Research Council under the European Union's Horizon 2020 research and innovation programme (Grant Agreement No. 758792, project GEODESI). S. G. S. is supported by the European Research Council under the European Community's Seventh Framework Programme (FP7/20072013 Grant Agreement No. 307934, NIRG project); he would also like to thank the Van Swinderen Institute for Particle Physics and Gravity for generous hospitality.

[1] R. Kallosh, A. Linde, and D. Roest, J. High Energy Phys. 11 (2013) 198.

[2] J. J. M. Carrasco, R. Kallosh, A. Linde, and D. Roest, Phys. Rev. D 92, 041301 (2015).

[3] A. Achúcarro, R. Kallosh, A. Linde, D.-G. Wang, and Y. Welling, J. Cosmol. Astropart. Phys. 04 (2018) 028.

[4] A. Achúcarro, V. Atal, C. Germani, and G. A. Palma, J. Cosmol. Astropart. Phys. 02 (2017) 013.

[5] P. Christodoulidis, D. Roest, and E. I. Sfakianakis, arXiv: 1803.09841.

[6] S. Garcia-Saenz, S. Renaux-Petel, and J. Ronayne, J. Cosmol. Astropart. Phys. 07 (2018) 057.

[7] T. Bjorkmo, Phys. Rev. Lett. 122, 251301 (2019).

[8] A. Hetz and G. A. Palma, Phys. Rev. Lett. 117, 101301 (2016). 
[9] A. Achúcarro and G. A. Palma, J. Cosmol. Astropart. Phys. 02 (2019) 041.

[10] G. Obied, H. Ooguri, L. Spodyneiko, and C. Vafa, arXiv: 1806.08362.

[11] P. Agrawal, G. Obied, P. J. Steinhardt, and C. Vafa, Phys. Lett. B 784, 271 (2018).

[12] E. Palti, Fortsch. Phys., 67, 1900037 (2019).

[13] S. Renaux-Petel and K. Turzyński, Phys. Rev. Lett. 117, 141301 (2016).

[14] S. Renaux-Petel, K. Turzyński, and V. Vennin, J. Cosmol. Astropart. Phys. 11 (2017) 006.

[15] T. Krajewski, K. Turzyński, and M. Wieczorek, Eur. Phys. J. C 79, 654 (2019).

[16] M. Cicoli, V. Guidetti, F. G. Pedro, and G. P. Vacca, J. Cosmol. Astropart. Phys. 12 (2018) 037.

[17] O. Grocholski, M. Kalinowski, M. Kolanowski, S. RenauxPetel, K. Turzyński, and V. Vennin, J. Cosmol. Astropart. Phys. 05 (2019) 008.

[18] A. R. Brown, Phys. Rev. Lett. 121, 251601 (2018).

[19] S. Mizuno and S. Mukohyama, Phys. Rev. D 96, 103533 (2017).

[20] T. Bjorkmo and M. C. D. Marsh, J. High Energy Phys. 04 (2019) 172.

[21] S. K. Garg and C. Krishnan, arXiv:1807.05193.

[22] H. Ooguri, E. Palti, G. Shiu, and C. Vafa, Phys. Lett. B 788, 180 (2019).

[23] J.-O. Gong, Int. J. Mod. Phys. D 26, 1740003 (2017).

[24] S. Groot Nibbelink and B. J. W. van Tent, Classical Quantum Gravity 19, 613 (2002).

[25] S. Cremonini, Z. Lalak, and K. Turzynski, J. Cosmol. Astropart. Phys. 03 (2011) 016.

[26] D. I. Kaiser, E. A. Mazenc, and E. I. Sfakianakis, Phys. Rev. D 87, 064004 (2013).

[27] Y. Wang, Commun. Theor. Phys. 62, 109 (2014).

[28] S. Renaux-Petel, C.R. Phys. 16, 969 (2015).

[29] Y. Akrami et al. (Planck Collaboration), arXiv:1905.05697.

[30] D. J. Mulryne and J. W. Ronayne, http://joss.theoj.org/ papers/10.21105/joss.00494 (2016).

[31] J. W. Ronayne and D. J. Mulryne, J. Cosmol. Astropart. Phys. 01 (2018) 023.
[32] M. Dias, J. Frazer, D. J. Mulryne, and D. Seery, J. Cosmol. Astropart. Phys. 12 (2016) 033.

[33] D. Seery, https://zenodo.org/record/61239.

[34] S. Butchers and D. Seery, J. Cosmol. Astropart. Phys. 07 (2018) 031.

[35] A. J. Tolley and M. Wyman, Phys. Rev. D 81, 043502 (2010).

[36] A. Achucarro, J.-O. Gong, S. Hardeman, G. A. Palma, and S. P. Patil, J. Cosmol. Astropart. Phys. 01 (2011) 030 .

[37] A. Achucarro, J.-O. Gong, S. Hardeman, G. A. Palma, and S. P. Patil, J. High Energy Phys. 05 (2012) 066.

[38] A. Achucarro, V. Atal, S. Cespedes, J.-O. Gong, G. A. Palma, and S. P. Patil, Phys. Rev. D 86, 121301 (2012).

[39] S. Garcia-Saenz and S. Renaux-Petel, J. Cosmol. Astropart. Phys. 11 (2018) 005.

[40] P. Creminelli, M. A. Luty, A. Nicolis, and L. Senatore, J. High Energy Phys. 12 (2006) 080.

[41] C. Cheung, P. Creminelli, A. L. Fitzpatrick, J. Kaplan, and L. Senatore, J. High Energy Phys. 03 (2008) 014.

[42] S. Garcia-Saenz, L. Pinol, and S. Renaux-Petel, arXiv: 1907.10403.

[43] X. Chen, M.-x. Huang, S. Kachru, and G. Shiu, J. Cosmol. Astropart. Phys. 01 (2007) 002.

[44] R. Holman and A. J. Tolley, J. Cosmol. Astropart. Phys. 05 (2008) 001.

[45] P. D. Meerburg, J. P. van der Schaar, and P. S. Corasaniti, J. Cosmol. Astropart. Phys. 05 (2009) 018.

[46] P. D. Meerburg, J. P. van der Schaar, and M. G. Jackson, J. Cosmol. Astropart. Phys. 02 (2010) 001.

[47] N. Agarwal, R. Holman, A. J. Tolley, and J. Lin, J. High Energy Phys. 05 (2013) 085.

[48] See Supplemental Material at http://link.aps.org/ supplemental/10.1103/PhysRevLett.123.201302 for a comparison between analytical and numerical bispectra, and details of our estimates of the trispectrum and higher-point correlation functions.

[49] T. Bjorkmo, R. Z. Ferreira, and M. C. D. Marsh, arXiv: 1908.11316.

[50] S. Weinberg, Phys. Rev. D 72, 043514 (2005). 